\title{
Stretchable and wearable colorimetric patches based on thermoresponsive plasmonic microgels embedded in a hydrogel film
}

\author{
Ayoung Choe ${ }^{1}$, Jeonghee Yeom ${ }^{1}$, Ravi Shanker', Minsoo P. Kim', Saewon Kang ${ }^{1}$ and Hyunhyub Ko $\mathbb{D}^{1}$
}

\begin{abstract}
Stimuli-responsive colorimetric sensors are promising for various industrial and medical applications due to the capability of simple, fast, and inexpensive visualization of external stimuli. Here we demonstrate a thermoresponsive, smart colorimetric patch based on a thermoresponsive plasmonic microgel embedded in a stretchable hydrogel film. To achieve a fast and efficient thermoresponsive color change, raspberry-shaped plasmonic microgels were fabricated by decorating gold nanoparticles (AuNPs) on poly(N-isopropylacrylamide) (PNIPAM) microgels, which exhibit reversible and strain-insensitive color shifts (between red and grayish violet) in response to a temperature change. The smart colorimetric patch containing a plasmonic microgels exhibits a significant extinction peak shift $(176 \mathrm{~nm})$ in a short time $(1 \mathrm{~s})$, with a temperature-sensing resolution of $0.2^{\circ} \mathrm{C}$. Moreover, the transition temperature of the plasmonic microgel can be finely tuned by additives and comonomers, so that the exquisite temperature visualization can be conducted over a wide temperature range of $25-40^{\circ} \mathrm{C}$ by assembling plasmonic microgel films with different transition temperatures into an array patch. For proof-of-concept demonstrations, a freestanding smart colorimetric patch was utilized as a spatial temperature scanner and a colorimetric thermometer for a thermoresponsive actuator, which is potentially applicable in smart, wearable sensors and soft robotics.
\end{abstract}

\section{Introduction}

Wearable health-care devices enable continuous health monitoring and offer instructive health-care information and record data for physical activity, electrocardiogram, body temperature, $\mathrm{pH}$ of sweat, and ultraviolet (UV) light ${ }^{1,2}$. Among these parameters, temperature is one of the main vital signs and is the most essential parameter for the diagnosis of the condition of the human body ${ }^{2}$. For example, body temperature provides information about health risks such as infections, inflammations, and antigenic reactions ${ }^{3}$. Different types of temperature sensors,

\footnotetext{
Correspondence: Hyunhyub Ko (hyunhko@unist.ac.kr)

${ }^{1}$ School of Energy and Chemical Engineering, Ulsan National Institute of Science and Technology (UNIST), Ulsan Metropolitan City 44919, Republic of Korea
}

These authors contributed equally: Ayoung Choe, Jeonghee Yeom including metal nanowires on a flexible substrate ${ }^{4}$, conductive fillers in an elastomeric matrix ${ }^{5}$, and metal nanoparticles in an organic semiconductor film ${ }^{6}$ have been developed to resolve the limitations of traditional mercury- and infrared-based thermometers such as rigidity, safety concerns, battery life, and cost issues. However, recently developed temperature sensors remain limited for wearable device applications due to complex fabrication steps, relatively bulky analytical instrumentation, and the requirement of an external power source.

Colorimetric sensors have the competitive advantages of being cost-effective and easy detection of stimuli without requiring complex and expensive analytical instruments ${ }^{7}$. There are many candidates for colorimetric sensors, such as photonic crystals ${ }^{8}$, thermochromic dye molecules ${ }^{9}$, and liquid crystals ${ }^{10}$. However, these technologies still show

\section{(c) The Author(s) 2018}

(c) (i) Open Access This article is licensed under a Creative Commons Attribution 4.0 International License, which permits use, sharing, adaptation, distribution and reproduction cc) in any medium or format, as long as you give appropriate credit to the original author(s) and the source, provide a link to the Creative Commons license, and indicate if changes were made. The images or other third party material in this article are included in the article's Creative Commons license, unless indicated otherwise in a credit line to the material. If material is not included in the article's Creative Commons license and your intended use is not permitted by statutory regulation or exceeds the permitted use, you will need to obtain permission directly from the copyright holder. To view a copy of this license, visit http://creativecommons.org/licenses/by/4.0/. 
limitations, including angle-dependent color differences, short fluorescence lifetime, and the need for an external electric field. On the other hand, plasmonic nanostructures can overcome these limitations and display a wide range of colors via the control of different plasmonic materials, geometries, and cluster structures ${ }^{11,12}$. The attractive colors of plasmonic nanostructures arise from a localized surface plasmon resonance (LSPR), which is the collective oscillation of conduction electrons in metallic nanoparticles when excited by an incident light ${ }^{13}$. Additionally, plasmon coupling-induced colors can show a remarkable shift when interparticle distances are finely tuned $^{14}$.

To control the interparticle distances for plasmonic nanoparticles under thermal stimuli, thermoresponsive colorimetric sensors have been developed by combining plasmonic nanoparticles with thermoresponsive poly $(N$ isopropylacrylamide) (PNIPAM) hydrogels ${ }^{15-17}$. The PNIPAM hydrogel has a lower critical solution temperature (LCST) of $32-34^{\circ} \mathrm{C}$, which is close to the temperature of the human body and exhibits a significant and reversible volume change (more than 18 times in the case of our PNIPAM microgels). Therefore, it has been widely used in many applications, such as actuators, biosensors, and drug-delivery vehicles ${ }^{18-21}$. Moreover, several studies have been carried out that employ the thermoresponsive nature of PNIPAM to fabricate colorimetric sensors by combining plasmonic nanoparticles with PNIPAM hydrogels. For example, Maji et al. ${ }^{15}$ showed the thermoresponsive color changes of PNIPAM-coated plasmonic nanoparticles, and Lim et al. ${ }^{16}$ designed plasmonic nanoparticles assembled on PNIPAM hydrogel colloids, which led to a visible response under external temperature changes. However, these solution-based plasmonic temperature sensors are inappropriate for utilization as a wearable sensor. Alternatively, Song et al. ${ }^{17}$ achieved thermoresponsive hydrogel architectures by decorating PNIPAM hydrogel colloids with plasmonic nanoparticles and incorporating the PNIPAM hydrogel colloids. However, the hydrogel-based sensor cannot be applied to a wearable patch-type sensor because large volume changes are necessary to implement thermoresponsive color shifts. In addition, a single sensor with one transition temperature cannot be used to detect a wide range of temperature. Therefore, for practical applications, challenges still remain to fabricate wearable colorimetric sensors with a wide range for temperature detection.

In this study, we demonstrate a stretchable and wearable colorimetric patch by embedding thermoresponsive plasmonic microgels inside a stretchable hydrogel film, which exhibits large and reversible color shifts in response to temperature changes without any volume change in the sensor itself. For a fast and efficient thermoresponsive color change, raspberry-shaped plasmonic microgels were fabricated by decorating gold nanoparticles (AuNPs) on PNIPAM microgels. The plasmonic microgels enabled a large thermoresponsive extinction peak shift $(176 \mathrm{~nm})$ in a short time (1 s) due to efficient plasmon coupling between outer AuNPs (through the large volumetric changes) and the fast phase transition behavior of PNIPAM microgels as a function of temperature. Furthermore, the incorporation of plasmonic microgels into the flexible polyacrylamide (PAAm) hydrogel film resolved the instability issue of colloidal solution and realized thermoresponsive color shifts without volumetric changes in the hydrogel film. When the flexible thin $(500 \mu \mathrm{m}$ thickness $)$ patches were in contact with hot objects of various shapes, the patches rapidly visualized (response time: $1 \mathrm{~s}$ ) the local temperature changes with a large extinction peak shift of $>170 \mathrm{~nm}$. By encapsulating the plasmonic microgel film with polydimethylsiloxane (PDMS) film, it can be stretched by up to $90 \%$ without showing a color change. Additionally, smart colorimetric array patches with varying color transition temperatures were fabricated by tuning the LCST of the plasmonic microgels, which exhibited a wide temperature-detection range $\left(29-40^{\circ} \mathrm{C}\right)$ and high resolution $\left(0.2^{\circ} \mathrm{C}\right)$. Due to the tunable LCST of plasmonic microgels, the thermoresponsive array patches can be applied as a colorimetric thermometer for thermoresponsive actuators to visualize the actuation temperature through color changes during continuous heating and cooling steps.

\section{Materials and methods \\ Materials}

$N$-isopropylacrylamide (NIPAM; 98\%), N,N'-methylenebisacrylamide (99\%), $\mathrm{HAuCl}_{4}$ (99.99\%), trisodium citrate dihydrate, 2,2-diethoxyacetophenone ( $>95 \%), 2-2^{\prime}$ azobis(2-methylpropionamidine) (98\%), acrylamide (AM; $\geq 99 \%$ ), butyl methacrylate (BMA; 99\%), sodium chloride $(\mathrm{NaCl} ; \geq 99.5 \%)$, and sodium dodecyl sulfate (SDS; $\geq 98.5 \%)$ were purchased from Sigma-Aldrich (MO, USA). Sylgard 184 was purchased from Dow Corning (MI, USA). All chemical compounds were used as received without further purification.

\section{Synthesis of PNIPAM microgel and AuNPs}

The PNIPAM microgels were synthesized following the work of Wei et al. ${ }^{22}$ NIPAM $(1613.1 \mathrm{mg})$ and $N, N^{\prime}$-methylenebisacrylamide $(0.1165 \mathrm{~g}, 5 \mathrm{~mol} \%$ relative to NIPAM) were dissolved in $145 \mathrm{~mL}$ of deionized water in a three-neck round-bottom flask with a reflux condenser followed by stirring and bubbling with nitrogen. After stirring the solution at $70^{\circ} \mathrm{C}$ for $40 \mathrm{~min}$, the polymerization was initiated by adding $5 \mathrm{~mL}$ of $0.03 \mathrm{M} \mathrm{2-2}$ azobis(2-methylpropionamide) aqueous solution. The solution was kept at $70^{\circ} \mathrm{C}$ for $3 \mathrm{~h}$. The resulting solution was purified by dialysis in a cellulose membrane with a 
molecular weight cutoff of $12-14 \mathrm{kDa}$ for $24 \mathrm{~h}$ to remove unreacted reagents. To fabricate the PNIPAM microgels with different LCSTs, the molar mixing ratio for NIPAM and comonomers (BMA or AM) was varied while the total number of moles of the monomers was held constant.

The AuNPs were synthesized by a kinetically controlled step-growth method ${ }^{23}$. Briefly, $150 \mathrm{~mL}$ of $2.2 \mathrm{mM}$ sodium citrate aqueous solution was heated to the boiling point under vigorous stirring. Then, $1 \mathrm{~mL}$ of $25 \mathrm{mM} \mathrm{HAuCl}_{4}$ aqueous solution was added. After $10 \mathrm{~min}$, the temperature was reduced to $90^{\circ} \mathrm{C}$ to inhibit any secondary nucleation. Then, $1 \mathrm{~mL}$ of the $\mathrm{HAuCl}_{4}$ solution was added and the mixture stirred for $30 \mathrm{~min}$; this step was repeated twice. Fifty-five milliliters of the solution were extracted, which was the product of each step. The solution was then diluted by adding $53 \mathrm{~mL}$ of deionized water and $2 \mathrm{~mL}$ of $60 \mathrm{mM}$ sodium citrate aqueous solution. The resulting solution was used as a seed solution for the next step. This process was repeated until the desired size for the AuNPs was obtained. The AuNPs were purified by centrifugation and redispersed in $0.9 \mathrm{mM}$ trisodium citrate aqueous solution. The plasmonic microgels were prepared by adding a specific amount of PNIPAM microgel solution to AuNP solution under moderate stirring for $1 \mathrm{~min}$.

\section{Fabrication of plasmonic microgels in bulk hydrogel}

Plasmonic microgels were embedded in PAAm hydrogels to counteract the precipitation problem. AM $(0.3554$ g), $N, N^{\prime}$-methylenebisacrylamide $(0.3622 \mathrm{~g})$, and 2,2-diethoxyacetophenone $(2.5 \mu \mathrm{L})$ were mixed into $1 \mathrm{~mL}$ of deionized water and nitrogen bubbled through the solution for $10 \mathrm{~min}$. The solution was added to the plasmonic microgel solution, which was also pretreated by bubbling with nitrogen, with a volumetric ratio of 1:4. The mixture was gently poured into the mold and the AM was polymerized under UV irradiation $(365 \mathrm{~nm}, 15 \mathrm{~W})$ for $17 \mathrm{~min}$.

\section{Fabrication of thermoresponsive color-changing actuator}

Polyethylene terephthalate (PET) films with a thickness of $25 \mu \mathrm{m}$ were cut into the shape of a human hand and used as the bottom layer. Plasmonic microgels in PAAm were prepared by a procedure similar to that used for plasmonic microgels in the bulk hydrogel; however, they were formed directly on the PET film by pouring the mixture of plasmonic microgels in the pre-PAAm solution into a PDMS frame $(0.5 \mathrm{~mm}$ thick), which was attached to the ends of the PET fingers, followed by the application of UV irradiation for $17 \mathrm{~min}$. Hydrogels were covered by thin PDMS film with a thickness of $150 \mu \mathrm{m}$ to prevent the evaporation of water. PNIPAM or P(NIPAMco-BMA) hydrogels with a thickness of $1 \mathrm{~mm}$ were cut into $5 \times 10 \mathrm{~mm}^{2}$ samples and attached to the PET film using double-sided tape. The critical temperatures were taken as the middle points of the regions exhibiting the sharpest thermoresponsive volume changes. The hydrogels were attached after removing the water on the surfaces of the hydrogels. To maximize the effect of the hydrogel volumetric changes on actuation, the doublesided tape was attached only at the ends of the hydrogels.

\section{Characterization}

The decoration of AuNPs on the PNIPAM microgels was observed using a scanning electron microscope (SEM, S-4800, Hitachi, Tokyo, Japan). The UV-visible (UV-Vis) spectra for the AuNPs and plasmonic microgels were measured using a UV-Vis-near-infrared spectrophotometer (Cary 5000, Agilent, CA, USA). The thermoresponsive color shift of the plasmonic microgels was measured by a spectroradiometer (PR-655, Photo Research, NY, USA). The dynamic light scattering (DLS) and zeta-potential (Nano ZS, Malvern Instruments, Malvern, UK) were measured to characterize the hydrodynamic diameter and surface charges of the AuNPs and the PNIPAM microgels. The temperature detection for the array patch was carried out on a thermal plate (TPCHS-C, Tokai Hit, Shizuoka, Japan).

\section{Results and discussion \\ Design and fabrication of the thermoresponsive colorimetric sensors}

For the design of the thermoresponsive colorimetric sensor, a raspberry-like architecture for the AuNPs on PNIPAM microgels (plasmonic microgels) in the PAAm hydrogel matrix was adopted, as illustrated in Fig. 1a. The raspberry-like architecture for the plasmonic microgels led to significantly large color changes caused by facile changes in the plasmonic mode between the uncoupled and coupled LSPR of the AuNPs ${ }^{14,24}$. The AuNPs on the PNIPAM microgels exhibited loosely packed structures at $24^{\circ} \mathrm{C}$ and densely packed assemblies at $50^{\circ} \mathrm{C}$, resulting in uncoupled and coupled plasmon modes at 24 and $50^{\circ} \mathrm{C}$, respectively. Figure 1b shows SEM images of the swollen and shrunken plasmonic microgels at 24 and $50{ }^{\circ} \mathrm{C}$ caused by thermoresponsive volume changes in the PNIPAM microgels. Moreover, to broaden the temperature detection range, the LCST of the PNIPAM microgels was controlled by adding additives, such as salt or surfactant, into the dispersion, or by copolymerizing NIPAM with hydrophilic or hydrophobic comonomers ${ }^{25-27}$. For practical application, the thermoresponsive colorimetric sensor can be integrated into a sensor array patch containing plasmonic microgel films with different transition temperatures, which can broaden the range of detectable temperature and visualize skin temperature more precisely when attached to the human skin (Fig. 1c).

For the attachment of AuNPs on the PNIPAM microgels, electrostatic attractive forces were utilized between the oppositely charged AuNPs and PNIPAM microgels. 

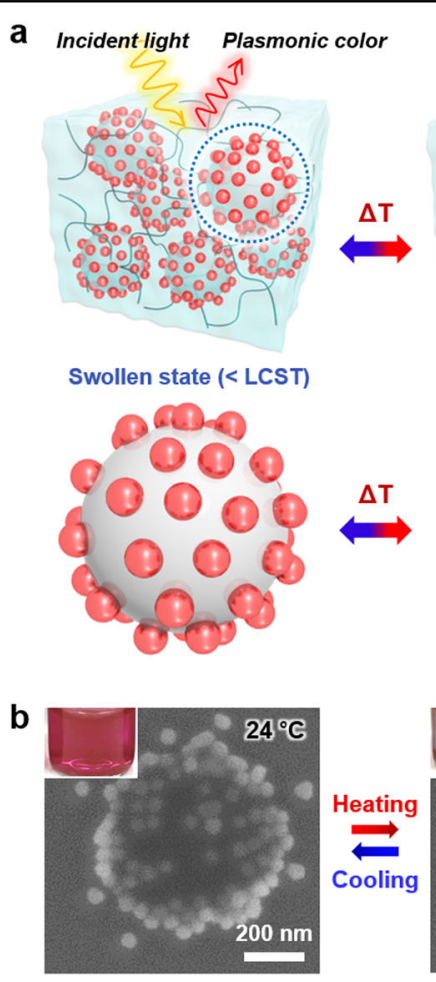

C
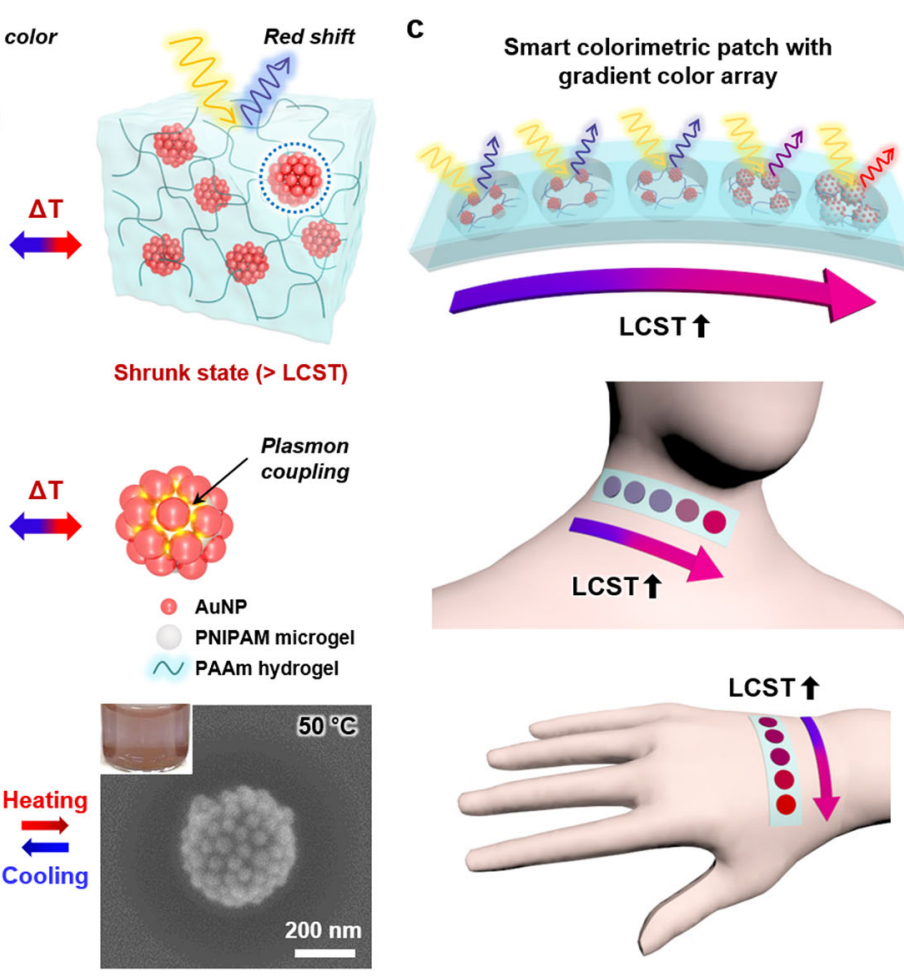

Fig. 1 The operating principle of a thermoresponsive colorimetric sensor. a Schematic illustration of the plasmonic microgels in the PAAm hydrogel under swollen and shrunk states. b SEM images of the plasmonic microgels with $51 \mathrm{~nm}$ AuNPs under the swollen state at $24^{\circ} \mathrm{C}$ (left) and under the shrunken state at $50^{\circ} \mathrm{C}$ (right); the inset images show pictures of the plasmonic microgel dispersions under each condition. $\mathbf{c}$ Schemes of the sensor array patches attached to human skin at different positions (neck and hand)

Negatively charged AuNPs with diameters in the range of 26-72 $\mathrm{nm}$ were synthesized by a kinetically controlled step-growth method (Supplementary Figure 1a) ${ }^{23}$. The UV-Vis spectra for the AuNP dispersion with different sizes show plasmonic absorbance bands at $524-543 \mathrm{~nm}$, where redshifts are observed with increasing AuNP size (Supplementary Figure 1b). The zeta-potential values indicate that the surface charges of AuNPs are negative with values between -34.6 and $-30.8 \mathrm{mV}$ and that they are not significantly influenced by the size difference (Supplementary Figure 1c). The net surface charge of AuNPs was negative due to stabilization of the AuNPs by the citrate molecules, with the large absolute values for the zeta-potential indicating that the AuNP dispersions are highly stable ${ }^{28}$. Positively charged PNIPAM microgels ( $550 \mathrm{~nm}$ in diameter) were synthesized by surfactant-free precipitation polymerization (Supplementary Figure $2 \mathrm{a})^{22}$. To create positive charges $(+14.5 \mathrm{mV})$ on the PNIPAM microgels, 2-2'-azobis(2-methylpropionamidine) was selected as a positively charged initiator (Supplementary Figure 2b). DLS analysis indicates that the surface area of the PNIPAM microgels changed greatly over sixfold, decreasing from 3.17 to $0.49 \mu^{2}$ for a decrease in diameter from 1004 to $396 \mathrm{~nm}$ according to the temperature change from 24 to $50^{\circ} \mathrm{C}$ (Supplementary Figure 2c). The dramatic changes in the surface area enabled effective control of the interparticle spacing between AuNPs. Here, to maximize the thermoresponsive color shift, the mixing ratio of AuNPs and PNIPAM microgels was optimized based on the SEM images and the peak shift in the UV-Vis spectra since neither an insufficient nor excess amount of AuNPs caused a strong color transition over the LCST. In the case of $51 \mathrm{~nm}$ AuNPs, the optimized mixing ratio for the AuNPs and PNIPAM microgels is 50:1 in volume because it shows the most densely coated AuNPs on PNIPAM microgels at $50^{\circ} \mathrm{C}$ and the biggest peak shift in the UV-Vis spectra (Supplementary Figure 3). An insufficient amount of AuNPs led AuNP assemblies on the PNIPAM microgels that were too sparse, which resulted in weak interparticle plasmon couplings over the LCST, with an excessive amount of AuNPs resulting in a coupled plasmon peak even before the temperature increment. Under the optimized mixing ratio for the AuNPs and PNIPAM microgels, the plasmonic microgels exhibit different thermoresponsive colors and UV-Vis peak shifts depending on the size of the AuNPs (Supplementary Figure 4$)^{14}$. Based on the analyses for the colors and UVVis spectra of different plasmonic microgel solutions at different temperatures, the plasmonic microgels with 51 $\mathrm{nm}$ AuNPs exhibit the largest color shift in response to 
temperature changes between 24 and $50^{\circ} \mathrm{C}$ and the least precipitation of microgels at $50^{\circ} \mathrm{C}$ (Supplementary Figure 4). Therefore, the plasmonic microgels with $51 \mathrm{~nm}$ AuNPs were selected for further analysis.

To fabricate a thermoresponsive colorimetric sensor with vivid color, the concentration of plasmonic microgel solution was increased by eight times. The concentrated plasmonic microgel solution was unstable because its zeta-potential value is close to zero (Supplementary Figure 5). This issue of an unstable plasmonic microgel solution was solved by embedding the plasmonic microgels in a bulk hydrogel matrix. PAAm was chosen as the bulk hydrogel matrix because it is not thermoresponsive, and, therefore, maintained a constant total volume with minimal interference with the volume changes of the plasmonic microgels. Thus, a PAAm hydrogel film (diameter, $5 \mathrm{~mm}$; thickness, $1 \mathrm{~mm}$ ) containing eight times concentrated plasmonic microgels was encapsulated between two thin $(150 \mu \mathrm{m}$ thickness $)$ PDMS films (Fig. 2a). The PAAm hydrogel film was able to hold the high concentration of plasmonic microgels in the matrix; therefore, the plasmonic microgels in hydrogel film exhibited a better stability during the repetitive heating/ cooling cycles and more vivid colors compared to the plasmonic microgel solution. Figure $2 \mathrm{~b}$ shows that the flexible plasmonic microgel film exhibits noticeable color shifts between red and grayish violet in response to the temperature changes between 24 and $50^{\circ} \mathrm{C}$ after $5 \mathrm{~s}$ heating and cooling processes. The high concentration of plasmonic microgels maximized the high thermal conductivity of the AuNPs, and the thin PAAm hydrogel films minimized the low thermal conductivity of the PAAm hydroge $^{29}$. The plasmonic microgel film exhibits a large peak shift of $176 \mathrm{~nm}$ (from 545 to $721 \mathrm{~nm}$ ) and highly stable and reversible peak shifts in the UV-Vis spectra after 10 heating/cooling cycles (Fig. 2c, d).

\section{Spatial temperature mapping and sensor stretchability}

A plasmonic microgel film was also fabricated as a form of large-area colorimetric patch with a large dimension over $10 \times 4 \mathrm{~cm}^{2}$, which enabled spatial temperature mapping of a complex shape, for example, by placing an object with the letters "UNIST" on the patch (Fig. 3a). When a heated object was applied to the patch, the colorimetric patch showed a rapid color shift in the local contact area. Figure $3 \mathrm{~b}$ shows the reversible thermoresponsive color shifts from red to grayish blue in the distinct contact area when aluminum objects with cylindrical or rectangular shapes (heated on $60^{\circ} \mathrm{C}$ hot plate) are in contact with the patches (Supplementary Figure 6a). The thermoresponsive color shift is identifiable even after $1 \mathrm{~s}$ of contact and the magnitude of the color shift increases with contact time (Fig. 3b). The colorimetric patch exhibits high flexibility with a bending radius of $1.5 \mathrm{~cm}$ and can be attached conformably and bent easily on the finger (Supplementary Figure 6b). Moreover, the thermoresponsive smart colorimetric patch was stretchable by encapsulating the plasmonic microgel film with a PDMS film. Figure 3c shows the stretchable patch with strain-insensitive colors, which can be stretched following the PDMS film without failure. The hydrogel-elastomer networks were built by covalent crosslinking of hydrogel polymers on the elastomer surface $^{30}$. The original size of the plasmonic hydrogel was $40 \times 15 \times 1 \mathrm{~mm}^{3}$, and it was stretched up to $90 \%$ in length in $3 \mathrm{~s}$ (Supplementary Video 1). As shown in Fig. 3d, the original plasmonic color and the thermally shifted color in the middle are not disturbed by stretching the sample up to $90 \%$ (from 4 to $7.6 \mathrm{~cm}$ in length).

The red, green, blue (RGB) values were obtained for every $10 \%$ increment in strain (Supplementary Figure 7). As the tensile strain was increased to $90 \%$, the relative changes in the red values for both unheated and heated regions exhibit negligible fluctuations compared to the red value for the unheated region at $0 \%$ strain (Fig. 3e). The linear-fitting graphs also showed slopes of $<0.003$, which indicated that the smart colorimetric patch had strain-insensitive colors. In addition, a very localized temperature change was monitored on an array film with dots of $2 \mathrm{~mm}$ in diameter (Supplementary Figure 8). The thermoresponsive colorimetric array film exhibits a localized color shift on the specific positions consisting of $1 \times$ $1,2 \times 2$, and $3 \times 3$ dots after contacting with a heated aluminum object (Supplementary Figure 8b-e).

\section{Wide detection range and high resolution of thermoresponsive colorimetric array patches}

One advantage of PNIPAM for temperature sensors is the facile control of the LCST by simply mixing with additives or adding comonomers ${ }^{25-27}$, which can broaden the range of detectable temperature. For example, the addition of salt is known to reduce the LCST of PNIPAM microgels by disrupting the hydration structure surrounding the PNIPAM polymer chains ${ }^{25}$. In the plasmonic microgels investigated here, the addition of $\mathrm{NaCl}$ up to $0.5 \mathrm{M}$ decreased the color transition temperature for the plasmonic microgel solution from 32 to $25^{\circ} \mathrm{C}$ (Supplementary Figure 9a). In contrast, the addition of a surfactant increases the LCST of the PNIPAM microgels by improving the solubility of the PNIPAM chains in water ${ }^{25}$. When the concentration of SDS was increased up to 4 $\mathrm{mM}$, the color transition temperature of the plasmonic microgel solution increases from 32 to $44^{\circ} \mathrm{C}$ (Supplementary Figure $9 \mathrm{~b}$ ). Figure 4a shows the color transition temperature of different plasmonic microgel solutions obtained by the aforementioned modifications as estimated by analyzing the colors of plasmonic microgel solutions at different temperatures (Supplementary 

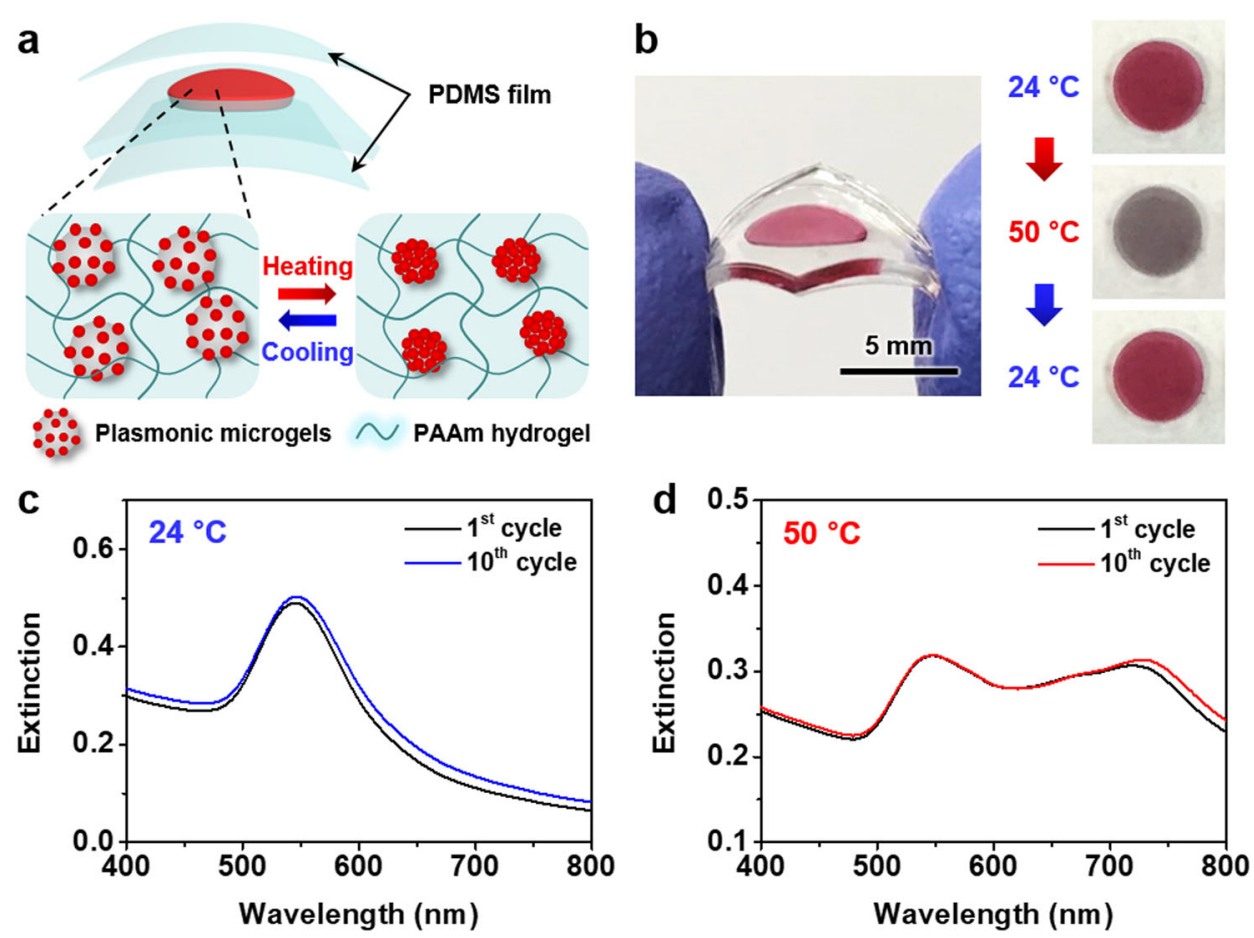

Fig. 2 Thermoresponsive color shifts of a plasmonic microgel film. a Schematic illustration of the plasmonic microgel films under the swollen and shrunk states encapsulated between PDMS films. $\mathbf{b}$ Pictures of the flexible plasmonic microgel films at $24^{\circ} \mathrm{C}$ and after heating and cooling for $5 \mathrm{~s}$. UV-Vis spectra for plasmonic microgel films after the first and tenth heating/cooling cycles obtained at $\mathbf{c} 24^{\circ} \mathrm{C}$ and $\mathbf{d} 50^{\circ} \mathrm{C}$

a

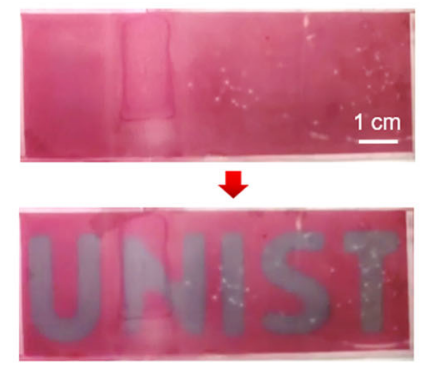

Response to a metal object $\left(60^{\circ} \mathrm{C}\right)$
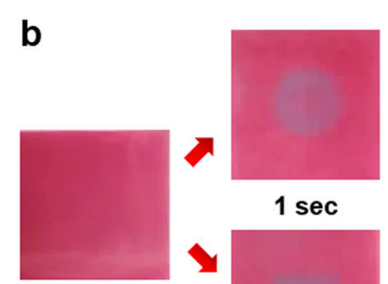

$24{ }^{\circ} \mathrm{C}$

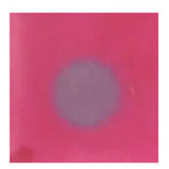

3 sec

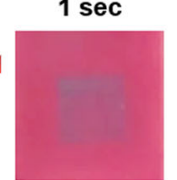

Response to a metal object $\left(60^{\circ} \mathrm{C}\right)$

5 sec
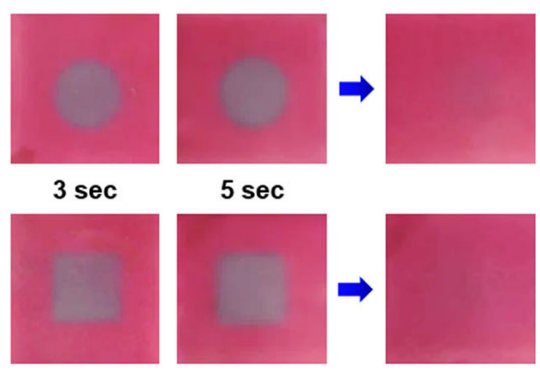

$24^{\circ} \mathrm{C}$
C

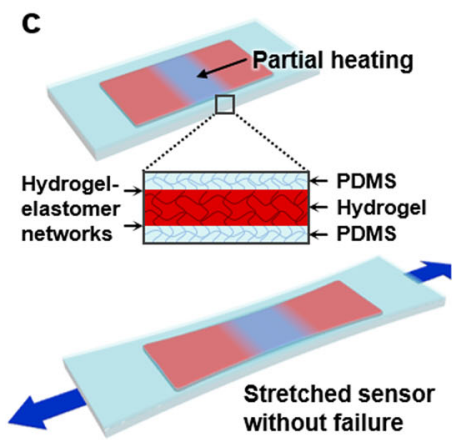

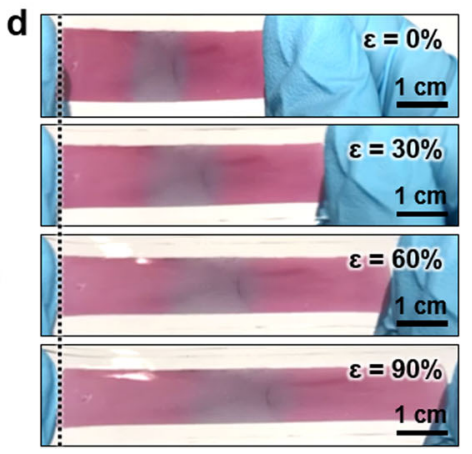

e

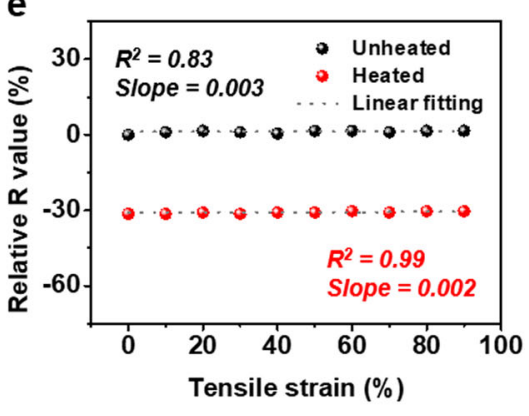

Fig. 3 Temperature mapping on a stretchable thermoresponsive colorimetric patch. a Pictures of temperature mapping for a letter-shaped aluminum object. b Pictures of a color-shifted film in contact with heated cylindrical and rectangular aluminum objects. c Schematic illustration of a stretching test under partial heating. $\mathbf{d}$ Pictures of stretchable patches with spatial color shift as a function of tensile strain. e Relative red value of unheated and heated regions compared to the red value of the unheated region at $0 \%$ strain as a function of tensile strain 
Figure 9). The color transition temperatures were determined to be $29,31.5,32,33.5$, and $37^{\circ} \mathrm{C}$ for the plasmonic microgel solutions with $0.2 \mathrm{M} \mathrm{NaCl}(\mathrm{N} 2), 0.1 \mathrm{M} \mathrm{NaCl}$ (N1), no additives (Ref), $1 \mathrm{mM}$ SDS (S1), and $2 \mathrm{mM}$ SDS (S2), respectively. The UV-Vis spectra for the plasmonic microgels in PAAm films with different types and concentrations of additives exhibit different peak shifts at 24 and $50{ }^{\circ} \mathrm{C}$ (Fig. $4 \mathrm{~b}$ ). The positions of the plasmon coupling peaks were determined by multiple peak fitting using a Gaussian function (Supplementary Figure 10). The plasmonic microgel films with higher transition temperatures exhibited a smaller shift in peak position. Specifically, the UV-Vis peak for $\mathrm{N} 2$ (transition temperature of $29^{\circ} \mathrm{C}$ ) shifts by over $200 \mathrm{~nm}$ (from 555 to $>758 \mathrm{~nm}$ ) while the $\mathrm{S} 2$ (transition temperature of $37^{\circ} \mathrm{C}$ ) exhibits a peak shift of only $9 \mathrm{~nm}$ (from 539 to $548 \mathrm{~nm}$ ) (Fig. 4c).

For the analysis of the thermoresponsive colorimetric array patch, the plasmonic microgel films with different transition temperatures (N2, N1, Ref, S1, and S2) were assembled into a single thermoresponsive array patch by sandwiching them between thin PDMS films. Then, the color changes of the colorimetric array patch were analyzed on a thermal plate $5 \mathrm{~s}$ after reaching the target temperature (Fig. 4d). The temperature resolution of the colorimetric sensor was examined by analyzing the RGB values of each pixel. Figure 4e shows the color change of the colorimetric array patch for temperature changes from 24 to $45^{\circ} \mathrm{C}$ with a $1{ }^{\circ} \mathrm{C}$ interval. The $\mathrm{N} 2$ pixel exhibited a color shift at the lowest temperature (approximately $30^{\circ} \mathrm{C}$ ), while the $\mathrm{S} 2$ pixel exhibited a color shift at the highest temperature (approximately $40^{\circ} \mathrm{C}$ ). The transition temperatures for the plasmonic microgel films were slightly higher than those for the plasmonic microgel solution with the same additives because the difference in the refractive indices affected the plasmon resonance band position ${ }^{31}$. In our work, the surrounding refractive index increased from 1.33 (water) to 1.47 $(\mathrm{PAAm})^{32}$ due to the embedding of the plasmonic microgels in the PAAm matrix, resulting in a redshift of the plasmon resonance band. Figure $4 \mathrm{f}$ shows the RGB values for the plasmonic microgel films with different transition temperatures measured in $1{ }^{\circ} \mathrm{C}$ increments. In the initial stage $\left(24^{\circ} \mathrm{C}\right)$, the $R$ values were dominant among three color values for all the pixels because the color of the plasmonic microgel films was close to red. For increasing temperature, the $R$ values decreased and the $B$ values increased, indicating that the colors changed to violet, which is a mixture of red and blue colors. The critical temperatures for the color change (vertical arrows in Fig. 4f), where the $R$ and $B$ values are close to each other, are $30,32,34,37$, and $40^{\circ} \mathrm{C}$ (from N2 to S2), which are highly correlated with the gradient transition temperatures in the thermoresponsive colorimetric array patch.
To further understand the property of the thermoresponsive colorimetric array patch, the colors of each pixel at different temperature conditions were measured from 24 to $45^{\circ} \mathrm{C}$ with a $1{ }^{\circ} \mathrm{C}$ interval by using a spectroradiometer (Fig. 4g). The $x$ and $y$ coordinates shifted from the red to violet region with increasing temperature. Specifically, the values for N2 shifted at the lowest temperature range $\left(25-30{ }^{\circ} \mathrm{C}\right)$ and the values of $\mathrm{S} 2$ shifted at the highest temperature range $\left(35-40^{\circ} \mathrm{C}\right)$. The detailed color spectra for the plasmonic microgel films also indicate a gradual increase of the intensity for the blue color approximately $450 \mathrm{~nm}$ due to the increasing temperature (Supplementary Figure 11). Moreover, our smart colorimetric patch has a high temperature resolution so that $0.2^{\circ} \mathrm{C}$ changes can be detected through an RGB analysis of the array patch (Supplementary Figure 12). In particular, as the temperature increased, the red values for sample S1 exhibit a high sensitivity of $-8.24{ }^{\circ} \mathrm{C}^{-1}$, with a high linearity of $R^{2}=0.99$ in the temperature range of 29$33^{\circ} \mathrm{C}$, via linear fitting, and the red values for sample S2 also exhibit a high sensitivity of $-8.76{ }^{\circ} \mathrm{C}^{-1}$, with a high linearity of $R^{2}=0.99$ in the temperature range of $33-40^{\circ} \mathrm{C}$ (Fig. 4h). For decreasing temperature, the sensors did not show distinct degradation of the sensitivity and linearity. The temperature-sensing capabilities of our colorimetric sensors can be favorably compared with previous reports. Supplementary Table 1 summarizes the materials used and the performance reported for colorimetric temperature sensors in recent studies ${ }^{9,15-17,29,33-37}$. Although many previous colorimetric temperature sensors based on plasmon coupling ${ }^{15-17,34,35}$ show large optical peak shifts (or color shifts), the response times range from several to tens of minutes, with most sensors in a solution state, which limits their practical applications in wearable sensors. Although other colorimetric temperature sensors built using photonic crystals ${ }^{29,33}$ and thermoresponsive dye molecules ${ }^{9,36,37}$ exhibit a shorter response time of $<1$ min, only a thermochromic liquid crystal-based sensor ${ }^{36}$ shows potential for application as a wearable temperature sensor. However, the thermochromic liquid crystal-based sensor has a lower temperature resolution (approximately $1{ }^{\circ} \mathrm{C}$ ) compared to our sensor $\left(0.2^{\circ} \mathrm{C}\right)$.

Furthermore, the thermoresponsive color shift of the thermoresponsive colorimetric patch is nearly impervious to environmental factors, such as environmental temperature, air flow, humidity, and light intensity. The thermoresponsive colorimetric patch built using plasmonic microgels shows negligible changes in RGB values on the thermal plate at constant temperature (30,35, and $40{ }^{\circ} \mathrm{C}$, respectively) when the environmental temperature, air flow, and relative humidity were changed (Supplementary Figure 13). The thermoresponsive colorimetric patch also exhibits minor fluctuations in RGB values under varying light intensities while the temperature of 


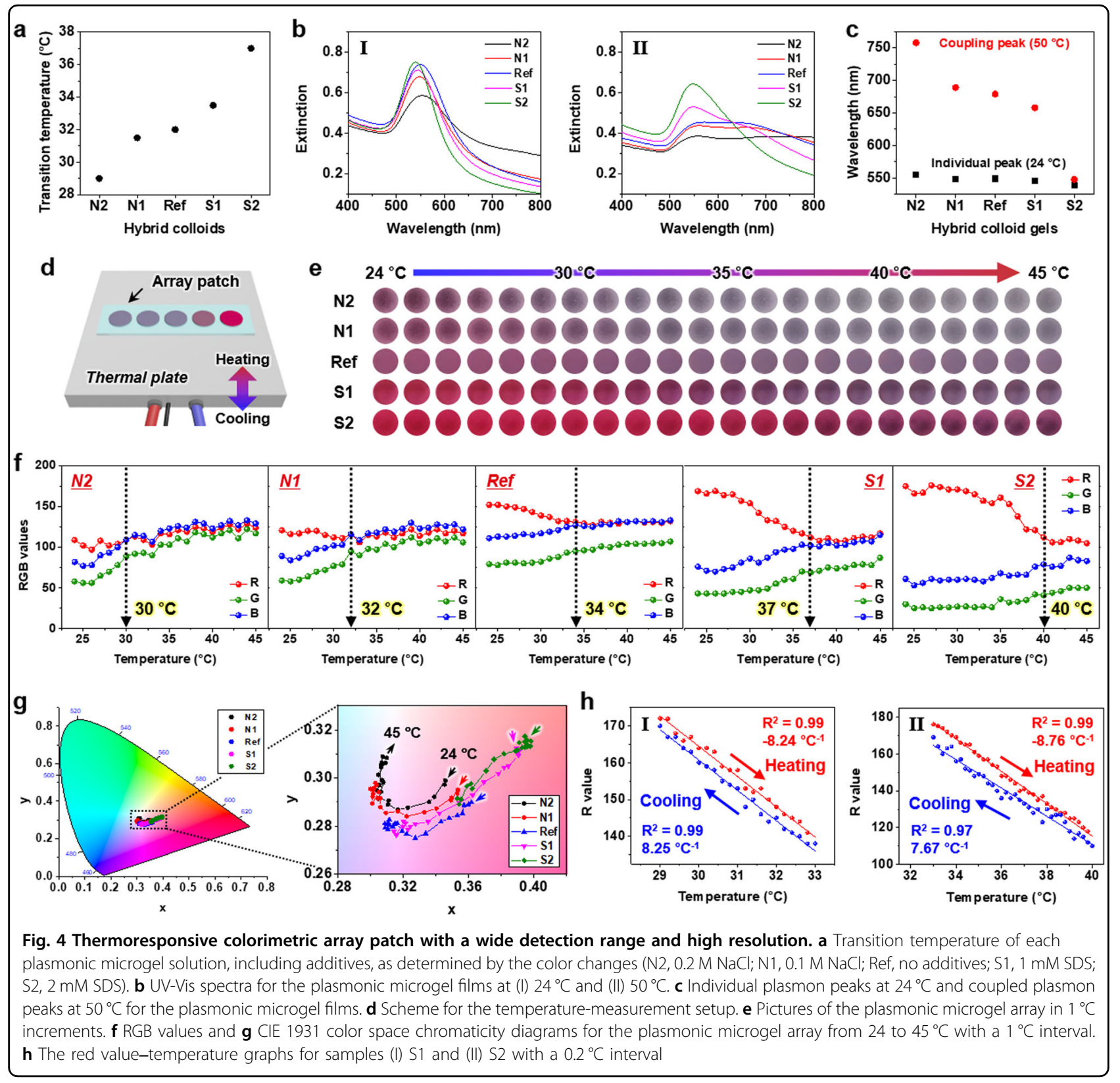

the thermal plate increased from 25 to $40{ }^{\circ} \mathrm{C}$ (Supplementary Figure 14).

\section{Colorimetric temperature visualization}

The sensor array patches visualized the temperature of human skin or the curved surface of an object by displaying different color patterns. When the thermoresponsive colorimetric array patch was placed onto a variety of surfaces, such as the human neck $\left(35.0^{\circ} \mathrm{C}\right.$ on the neck), wrist and back of the hand $\left(34.1^{\circ} \mathrm{C}\right.$ on the wrist, $21.6^{\circ} \mathrm{C}$ on the back of the hand after washing), and surface of a beaker filled with water $\left(29.0^{\circ} \mathrm{C}\right.$ on the beaker surface), the array patch displays different color patterns at each temperature (Fig. 5a-c). The temperature of human skin and a water-filled beaker were confirmed by using traditional thermometers (Supplementary Figure 15). This result verifies that it is possible to use the proposed thermoresponsive colorimetric patch as a wearable and attachable temperature sensor. Additionally, the combination of a thermoresponsive sensor with actuator technologies enables both optical output signals and mechanical actuation in response to temperature changes, which makes it possible to monitor temperature in real time via thermoresponsive actuation ${ }^{38,39}$. In this study, we developed a thermoresponsive smart hand by assembling the plasmonic microgel films on fingertips for 
a
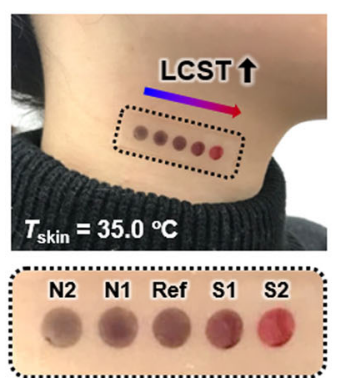
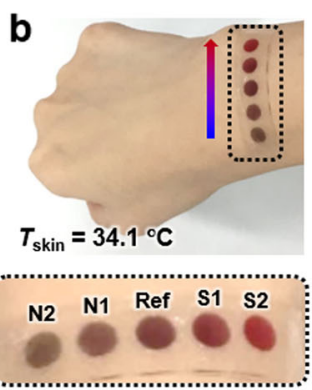

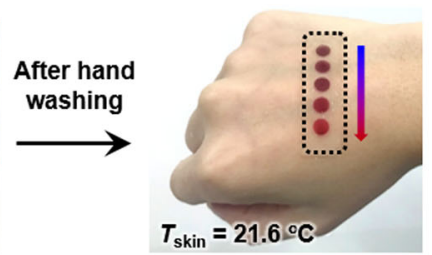

N2 N1 Ref $\mathrm{S} 1 \mathrm{~S} 2$
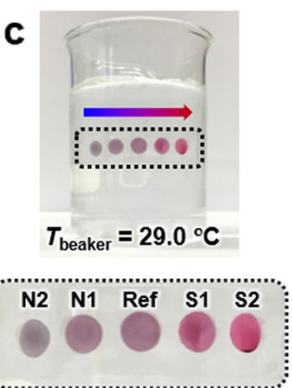

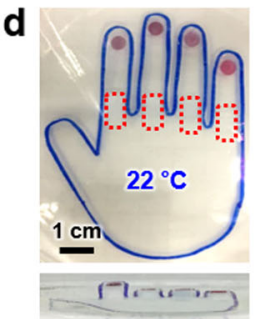

Thermoresponsive color change of fingertip (Top view)

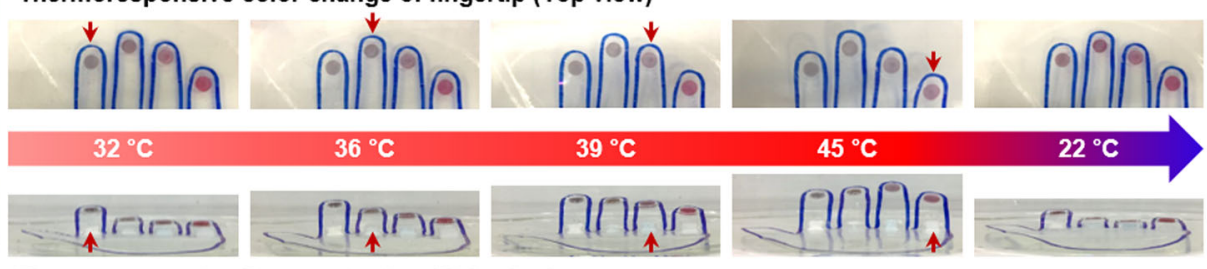

Thermoresponsive finger actuation (Side view)

Fig. 5 Colorimetric visualization of the thermoresponsive smart colorimetric patch. Pictures of the color array patch attached to the. $\mathbf{a}$ neck, $\mathbf{b}$ hand, and $\mathbf{c}$ a beaker filled with water. (The pictures were taken $5 \mathrm{~s}$ after attachment of the patches.) $\mathbf{d}$ Pictures of the hand-shaped actuator, composed of a plasmonic co-microgel film on the fingertips and co-PNIPAM films on the finger joints, placed in a water bath at different temperatures for $1 \mathrm{~min}$. (The red-dotted boxes indicate the positions of the co-PNIPAM films, and the red arrows indicate the specific finger exhibiting the color change and actuation.)

thermoresponsive color output and PNIPAM films on finger joints (red-dotted rectangles in the figure) for thermoresponsive actuation (Fig. 5d). In Fig. 5d, PNIPAM films were attached to the joints of a $25 \mu \mathrm{m}$-thick handshaped PET film for double-layer structured actuators, which showed behavior analogous to that of finger joints. For efficient heat transfer to the smart hand, the experiment was performed in a water bath. In this system, the concentration of additives in PNIPAM hydrogels can be altered by solvent dilution when the PNIPAM hydrogels are soaked in a liquid medium. Therefore, the LCST tuning was carried out by copolymerization of the PNIPAM to overcome the stability issue for additivecontaining PNIPAM hydrogels.

The LCST of the PNIPAM hydrogels can be increased or decreased by copolymerizing NIPAM with hydrophilic or hydrophobic comonomers, respectively ${ }^{25-27}$. To achieve a lower LCST, BMA was selected since the butyl groups in BMA impart more hydrophobic characteristics to the thermoresponsive hydrogel ${ }^{26}$. In contrast, $\mathrm{AM}$ was selected for the synthesis of PNIPAM hydrogels with higher LCST, since it introduces more hydrophilic properties to the hydrogels ${ }^{27}$. Here the same attachment principle was employed based on electrostatic interaction between copolymerized PNIPAM microgels and AuNPs since $\mathrm{P}$ (NIPAM-co-BMA) and P(NIPAM-co-AM) microgels were obtained with positive surface charges (Supplementary Figure 16). To broaden the range of temperature detection, PNIPAM microgels with either
BMA or AM as comonomers were synthesized with different ratios of NIPAM to BMA or AM. Based on the endothermic peaks identified from the differential scanning calorimetry thermograms, it was confirmed that the transition temperature of the copolymerized plasmonic microgels (plasmonic co-microgels) was tuned over a wide range of $28.0-44.8^{\circ} \mathrm{C}$ (Supplementary Figure 17). For the thermoresponsive actuation of fingers at different temperatures, the copolymerized PNIPAM bulk films (coPNIPAM films) with different transition temperatures (approximately $34.0,35.8,38.9$, and $43.5^{\circ} \mathrm{C}$ ) were fabricated with $20,15,5$, and $0 \mathrm{~mol} \%$ of BMA, respectively (Supplementary Figure 18). The co-PNIPAM films with different transition temperatures were attached onto the finger joints in the order of increasing transition temperature from the index finger to the little finger. Here, to match the transition temperature of the plasmonic comicrogel film with that of the co-PNIPAM film on the same finger, the plasmonic co-microgel films (BMA20, PNIPAM, AM9.1, and AM16.6) showing different transition temperatures (approximately 31.9, 34.9, 40.2, and $44.8^{\circ} \mathrm{C}$, respectively, Supplementary Figure $17 \mathrm{a}$ ) were attached onto the fingertips (from the index finger to the little finger). The plasmonic co-microgel and the coPNIPAM film with the same comonomer condition showed different transition temperatures because different hydrogel thicknesses and NIPAM concentrations also affected the transition temperatures. The co-PNIPAM film was twice the thickness of the plasmonic co-microgel 
film $(500 \mu \mathrm{m}$ thick), with NIPAM concentrations of 0.95 and $6.4 \times 10^{4} \mathrm{~mol} \mathrm{~g}^{-1}$ used for the synthesis of the microgel and hydrogel, respectively, resulting in different transition temperatures at approximately 34 and $32{ }^{\circ} \mathrm{C}^{40}$.

Figure $5 \mathrm{~d}$ shows that the smart hand is in an open position and that the fingertips are red color in water at $22^{\circ} \mathrm{C}$. When the water temperature was increased to approximately $32^{\circ} \mathrm{C}$, the color of the index fingertip changed from red to grayish violet and the index finger began to bend because the temperature was close to the transition temperature of the plasmonic co-microgel and co-PNIPAM films on both the fingertip and joint. As the temperature was gradually increased further, the colors of the fingertips changed and finger bending occurred sequentially in the order of middle, ring, and little fingers due to the sequential increase in the transition temperature of the plasmonic co-microgel and co-PNIPAM films on these fingers. In water at $45^{\circ} \mathrm{C}$, all of the fingers bent and the colors at the fingertips were grayish violet. When the water temperature was cooled back to $22^{\circ} \mathrm{C}$, the smart hand returned to the open position and the fingertip colors changed back to red. The fingertip of the smart hand exhibited rapid (approximately $5 \mathrm{~s}$ ) color changes but a relatively slow (approximately $1 \mathrm{~min}$ ) finger-bending motion in response to both heating and cooling processes due to the relatively large size of the hydrogel actuators compared to the thermoresponsive colorimetric sensors. The tunable critical temperatures for both color patches and actuators in the smart hand enabled sequential responses by way of color changes and movements of fingers in response to varying temperature, which is beneficial for smart sensors, actuators, and soft robotics with various geometries ${ }^{21,38}$.

\section{Conclusions}

In summary, we developed a thermoresponsive, smart colorimetric patch based on raspberry-shaped plasmonic microgels encapsulated in a stretchable PAAm hydrogel film. For a fast and efficient thermoresponsive color change, the raspberry-shaped plasmonic microgels were fabricated by decorating AuNPs on PNIPAM microgels, which induced a large color change via reversible tuning of the plasmon couplings between AuNPs when the thermoresponsive PNIPAM microgels reversibly swelled and shrunk in response to thermal stimuli. The PAAm hydrogel film stabilized the plasmonic microgels against aggregation and held the high concentration of plasmonic microgels in the matrix, which enabled stronger plasmonic couplings in the plasmonic microgel film compared to a plasmonic microgel solution. The plasmonic microgel films resulted in vivid color changes with high reversibility and stretchability. Furthermore, the transition temperature was easily tuned over the range of $25.0-44.8^{\circ} \mathrm{C}$ by adding salt, surfactant, or copolymerizing with comonomers, which enabled thermoresponsive array patches to cover a wide temperature-detection range of $25-40^{\circ} \mathrm{C}$. The freestanding thin-film structure of the colorimetric patches resulted in a rapid and dramatic color change from red to grayish violet and a shift in the extinction peak by $176 \mathrm{~nm}$ in $1 \mathrm{~s}$, with the realization of a temperature-sensing resolution of $0.2^{\circ} \mathrm{C}$, which is higher than that obtained for previous colorimetric temperature sensors based on plasmonic coupling. As a proof-of-concept application, we demonstrated a smart hand that exhibited sequential responses in the way of color changes and movements of fingers in response to changes in the environmental temperature. Additional combinations of various types of plasmonic nanoparticles and hydrogel microstructures can be explored in the future to broaden the applications of smart colorimetric patches for smart sensors and actuators.

\section{Acknowledgements}

This work was supported by the National Research Foundation of Korea (2015R1A2A1A10054152 and 2017M1A2A2087833) and the Ministry of Trade, Industry and Energy of Korea (10067082).

Conflict of interest

The authors declare no that they have no conflict of interest.

\section{Publisher's note}

Springer Nature remains neutral with regard to jurisdictional claims in published maps and institutional affiliations.

Supplementary Information is available for this paper at https://doi.org/ 10.1038/s41427-018-0086-6.

Received: 20 April 2018 Revised: 7 August 2018 Accepted: 14 August 2018. Published online: 20 September 2018

\section{References}

1. Yamamoto, Y. et al. Printed multifunctional flexible device with an integrated motion sensor for health care monitoring. Sci. Adv. 2, e1601473 (2016).

2. Nakata, S., Arie, T., Akita, S. \& Takei, K. Wearable, flexible, and multifunctional healthcare device with an ISFET chemical sensor for simultaneous sweat pH and skin temperature monitoring. ACS Sens. 2, 443-448 (2017).

3. Evans, S. S., Repasky, E. A. \& Fisher, D. T. Fever and the thermal regulation of immunity: the immune system feels the heat. Nat. Rev. Immunol. 15, 335 (2015).

4. Chen, Y., Lu, B., Chen, Y. \& Feng, X. Breathable and stretchable temperature sensors inspired by skin. Sci. Rep. 5, 11505 (2015).

5. Trung, T. Q., Ramasundaram, S., Hwang, B. U. \& Lee, N. E. An all-elastomeric transparent and stretchable temperature sensor for body-attachable wearable electronics. Adv. Mater. 28, 502-509 (2016).

6. Ren, $X$. et al. A low-operating-power and flexible active-matrix organic-transistor temperature-sensor array. Adv. Mater. 28, 4832-4838 (2016).

7. Rakow, N. A. \& Suslick, K. S. A colorimetric sensor array for odour visualization. Nature 406, 710 (2000).

8. Wang, H. \& Zhang, K.-Q. Photonic crystal structures with tunable structure color as colorimetric sensors. Sensors 13, 4192-4213 (2013).

9. Kim, G. et al. Spatially pressure-mapped thermochromic interactive sensor. Adv. Mater. 29, 1606120 (2017).

10. Tokunaga, S. et al. Electrophoretic deposition for cholesteric liquid-crystalline devices with memory and modulation of reflection colors. Adv. Mater. 28 4077-4083 (2016).

11. González, A., Noguez, C., Beránek, J. \& Barnard, A. Size, shape, stability, and color of plasmonic silver nanoparticles. J. Phys. Chem. C 118, $9128-9136$ (2014). 
12. Kristensen, A. et al. Plasmonic colour generation. Nat. Rev. Mater. 2, 16088 (2016).

13. Willets, K. A. \& Van Duyne, R. P. Localized surface plasmon resonance spectroscopy and sensing. Annu. Rev. Phys. Chem. 58, 267-297 (2007).

14. Ghosh, S. K. \& Pal, T. Interparticle coupling effect on the surface plasmon resonance of gold nanoparticles: from theory to applications. Chem. Rev. 107, 4797-4862 (2007).

15. Maji, S., Cesur, B., Zhang, Z., De Geest, B. G. \& Hoogenboom, R. Poly (Nisopropylacrylamide) coated gold nanoparticles as colourimetric temperature and salt sensors. Polym. Chem. 7, 1705-1710 (2016).

16. Lim, S., Song, J. E., La, J. A. \& Cho, E. C. Gold nanospheres assembled on hydrogel colloids display a wide range of thermoreversible changes in optical bandwidth for various plasmonic-based color switches. Chem. Mater. 26 3272-3279 (2014)

17. Song, J. E. \& Cho, E. C. Dual-responsive and multi-functional plasmonic hydrogel valves and biomimetic architectures formed with hydrogel and gold nanocolloids. Sci. Rep. 6, 34622 (2016).

18. Mura, S., Nicolas, J. \& Couvreur, P. Stimuli-responsive nanocarriers for drug delivery. Nat. Mater. 12, 991 (2013).

19. Sigolaeva, L. V. et al. Dual-stimuli-sensitive microgels as a tool for stimulated spongelike adsorption of biomaterials for biosensor applications. Biomacromolecules 15, 3735-3745 (2014).

20. Lee, $H$. et al. Octopus-inspired smart adhesive pads for transfer printing of semiconducting nanomembranes. Adv. Mater. 28, 7457-7465 (2016).

21. Ko, H. \& Javey, A. Smart actuators and adhesives for reconfigurable matter. Acc. Chem. Res. 50, 691-702 (2017).

22. Wei, J. et al. Investigation of cell behaviors on thermo-responsive PNIPAM microgel films. Colloids Surf. B 132, 202-207 (2015).

23. Bastús, N. G., Comenge, J. \& Puntes, V. Kinetically controlled seeded growth synthesis of citrate-stabilized gold nanoparticles of up to $200 \mathrm{~nm}$ : size focusing versus Ostwald ripening. Langmuir 27, 11098-11105 (2011).

24. Guo, L., Xu, Y., Ferhan, A. R., Chen, G. \& Kim, D.-H. Oriented gold nanoparticle aggregation for colorimetric sensors with surprisingly high analytical figures of merit. J. Am. Chem. Soc. 135, 12338-12345 (2013).

25. Van Durme, K., Rahier, H. \& Van Mele, B. Influence of additives on the thermoresponsive behavior of polymers in aqueous solution. Macromolecules $\mathbf{3 8}$ 10155-10163 (2005).

26. Das, M., Sanson, N., Fava, D. \& Kumacheva, E. Microgels loaded with gold nanorods: photothermally triggered volume transitions under physiological conditions. Langmuir 23, 196-201 (2007).
27. Cheng, J., Shan, G. \& Pan, P. Temperature and pH-dependent swelling and copper (II) adsorption of poly ( $\mathrm{N}$-isopropylacrylamide) copolymer hydrogel. RSC Adv. 5, 62091-62100 (2015).

28. Kim, T., Lee, K., Gong, M. \& Joo, S.-W. Control of gold nanoparticle aggregates by manipulation of interparticle interaction. Langmuir $\mathbf{2 1}$ 9524-9528 (2005).

29. Yan, $Y$. et al. Plasmonic nanoparticles tuned thermal sensitive photonic polymer for biomimetic chameleon. Sci. Rep. 6, 31328 (2016).

30. Yuk, H., Zhang, T., Parada, G. A., Liu, X. \& Zhao, X. Skin-inspired hydrogel-elastomer hybrids with robust interfaces and functional microstructures. Nat. Commun. 7, 12028 (2016).

31. Mock, J. J., Smith, D. R. \& Schultz, S. Local refractive index dependence of plasmon resonance spectra from individual nanoparticles. Nano Lett. 3, 485-491 (2003).

32. Franklin, J. \& Wang, Z. Y. Refractive index matching: a general method for enhancing the optical clarity of a hydrogel matrix. Chem. Mater. 14, 4487-4489 (2002).

33. Chen, M., Zhou, L., Guan, Y. \& Zhang, Y. Polymerized microgel colloidal crystals: photonic hydrogels with tunable band gaps and fast response rates. Angew. Chem. Int. Ed. 52, 9961-9965 (2013).

34. Liu, Y., Han, X., He, L. \& Yin, Y. Thermoresponsive assembly of charged gold nanoparticles and their reversible tuning of plasmon coupling. Angew. Chem. Int. Ed. 51, 6373-6377 (2012)

35. Ding, T. et al. Light-induced actuating nanotransducers. Proc. Natl Acad. Sci. USA 113, 5503-5507 (2016).

36. Gao, L. et al. Epidermal photonic devices for quantitative imaging of temperature and thermal transport characteristics of the skin. Nat. Commun. $\mathbf{5}$, 4938 (2014).

37. Chae, S., Lee, J. P. \& Kim, J. M. Mechanically drawable thermochromic and mechanothermochromic polydiacetylene sensors. Adv. Funct. Mater. 26 1769-1776 (2016)

38. Jin, Y.-J. et al. Phase-change hybrids for thermo-responsive sensors and actuators. NPG Asia Mater. 6, e137 (2014).

39. Xing, H., Li, J., Shi, Y., Guo, J. \& Wei, J. Thermally driven photonic actuator based on silica opal photonic crystal with liquid crystal elastomer. ACS Appl. Mater. Interfaces 8, 9440-9445 (2016).

40. Otake, K., Inomata, H., Konno, M. \& Saito, S. Thermal analysis of the volume phase transition with N-isopropylacrylamide gels. Macromolecules 23, 283-289 (1990). 